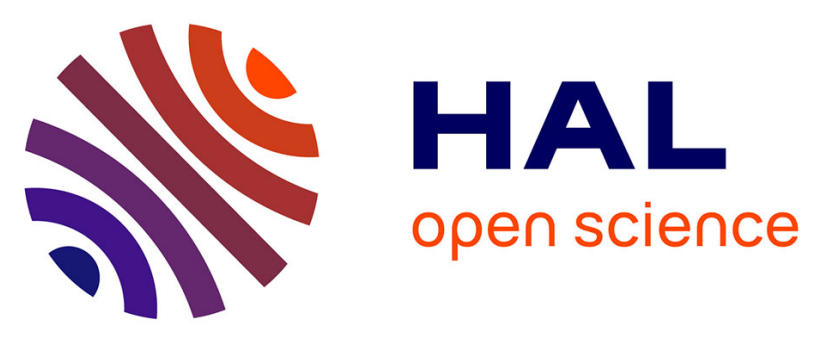

\title{
Stability and response analysis of a pipe with internal flow analyzed with an uncertain computational model
}

\author{
T. G. Ritto, Christian Soize, A. F. Rochinha, R. Sampaio
}

\section{To cite this version:}

T. G. Ritto, Christian Soize, A. F. Rochinha, R. Sampaio. Stability and response analysis of a pipe with internal flow analyzed with an uncertain computational model. COMPDYN 2013, 4th ECCOMAS Thematic Conference on Computational Methods in Structural Dynamics and Earthquake Engineering, Jun 2013, Kos Island, Greece. pp.1-14. hal-00806404

\section{HAL Id: hal-00806404 https://hal.science/hal-00806404}

Submitted on 30 Mar 2013

HAL is a multi-disciplinary open access archive for the deposit and dissemination of scientific research documents, whether they are published or not. The documents may come from teaching and research institutions in France or abroad, or from public or private research centers.
L'archive ouverte pluridisciplinaire HAL, est destinée au dépôt et à la diffusion de documents scientifiques de niveau recherche, publiés ou non, émanant des établissements d'enseignement et de recherche français ou étrangers, des laboratoires publics ou privés. 


\title{
STABILITY AND RESPONSE OF A PIPE WITH INTERNAL FLOW ANALYZED WITH AN UNCERTAIN COMPUTATIONAL MODEL
}

\author{
T.G. Ritto ${ }^{1}$, C. Soize ${ }^{2}$, F.A. Rochinha ${ }^{1}$, and Rubens Sampaio ${ }^{3}$ \\ ${ }^{1}$ Federal University of Rio de Janeiro, Dept of Mechanical Engineering \\ Centro de Tecnologia, Ilha do Fundão, 21945-970, Rio de Janeiro, Brazil \\ e-mail: tritto@mecanica.ufrj.br, faro@mecanica.ufrj.br \\ ${ }^{2}$ Université Paris-Est, Laboratoire de Modélisation et Simulation Multi-Echelle \\ MSME UMR 8208 CNRS, 5bd Descartes, 77454 Marne-la-Vallée Cedex 2, France \\ e-mail: christian.soize@univ-paris-est.fr \\ ${ }^{3}$ PUC-Rio, Dept of Mechanical Engineering \\ Rua Marquês de São Vicente, 225, 22453-900, Rio de Janeiro, Brazil \\ e-mail: rsampaio@puc-rio.br
}

Keywords: stochastic dynamics, fluid-structure model uncertainty, stability analysis, uncertainty quantification

\begin{abstract}
This paper treats the problem of pipes conveying fluid, which has several engineering applications, such as micro-systems and drill-string dynamics. The aim of this work is twofold: (1) propose a stochastic model for the fluid-structure interaction considering modeling errors and (2) analyze the stability of the stochastic system. The Euler-Bernoulli model is used to model the pipe and the plug flow model is used to take into account the presence of the internal flow. The resulting differential equation is discretized by means of the finite element method and a reduced-order model is constructed with the normal modes of the beam model. A variation of the nonparametric probabilistic approach is used to model uncertainties in the fluid-structure interaction, since this approach is able to take into account modeling errors. The numerical results show how the random response of the system changes for different levels of uncertainties. The probability of instability is also computed for different levels of uncertainties.
\end{abstract}




\section{INTRODUCTION}

Slender flexible tubes with internal flow or pipes conveying fluids are present in a number of applications ranging from micro-systems to biological devices, drill-strings or heat exchangers [1, 2, 3]. Typically, the standard configuration is a straight tube mounted over supports carrying a steady flow of constant velocity $U$ [4]. Ambient perturbations might entail low amplitude vibrations of the structure around the standard configuration if the velocity is kept below a certain threshold. Above the critical speed, the coupled system formed by the structure and the flow might undergo large vibrations and complex nonlinear dynamical response. To understand this unstable behavior [5] becomes instrumental for improving the design of the systems and mitigate damage effects. Besides, this large diversity in the vibrations response renders this problem quite attractive for theoretical and numerical studies.

Computer models are nowadays widespreadly used in the design and analysis of standard engineering systems. Many noncritical decisions are taken based on computational simulations. Despite the consolidation of powerful and reliable methods leading to small numerical errors, the extension of this common practice to more critical systems is hindered by the presence of inevitable uncertainties associated to the modeling. Fluctuations around nominal values of parameters, initial and boundary conditions, or production tolerances might entail, after a number of nonlinear calculations, a large variation on the output of the simulations. So, in order to improve the reliability of predictions, those uncertainties must be taken into account. Here, this is carried out within a probabilistic framework in which physical quantities involved in the modeling are represented by random variables or fields. Design criteria are then based on failure probabilities and reliability analysis [6].

On the initial stages of the analysis, low order models considering simplified physics are employed. The use of simple models makes feasible to analyze a significant number of scenarios. Complying with that, the flexible tubes are modeled here as Euler-Bernoulli beams and the internal fluid motion is described through a one-space dimension plug flow model, such that the resulting coupled model consists on a one-dimensional linear partial differential equation parametrized by the flow speed $U$. The deterministic analysis of the stability of this system has been studied [7], thus we propose an analysis of the stability of the stochastic system. In [8] the stochastic stability analysis is done considering stochastic time varying loads, which differs from the present analysis, in which the system is uncertain.

The coupling is responsible for the existence of unstable modes of the system and, therefore, plays a central role in the present analysis. In order to endow the model with an improved capacity of describing the fluid-structure coupling, a probabilistic model relying upon the nonparametric approach for uncertainties introduced in [9] is proposed. That approach has been applied in many different areas [1, 10, 11] to cope with uncertainties arising from different sources along the modeling process.

This nonparametric probabilistic approach, to be described in more details later, consists in deriving for the reduced-order model of the original problem, random matrices corresponding to a probabilistic density function obtained with the available information through the principle of maximum entropy [12]. Here, two matrices are obtained associated to damping and stiffness engendered by the flow. They replace the ones derived based on simplifications of the coupled system kinematics. Thus, the stability analysis considering randomness relies on solving a stochastic eingenvalue problem. The eingenvalues are now random variables and the stability is dictated by the probability of their sign to be positive. A number of results is presented with emphasis on the statistics characterization of the eingenvalues. 
This paper is organized as follows: the deterministic system is depicted in Sections 2, 3,4 and 5. where the equations, the dimensionless quantities and the stability analysis are presented. The probabilistic model is developed in Section 6. Finally, the numerical results are shown in Section 7 and the concluding remarks are made in Section 8 .

\section{DETERMINISTIC MODEL}

Figure 1 sketches the system considered in the analysis.

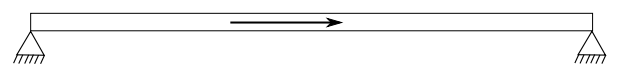

Figure 1: Sketch of the system considered in the analysis (the arrow represents the internal fluid flow).

Using the Euler-Bernoulli beam theory, the partial differential equation governing the dynamics of the structure is written as [13]:

$$
m \frac{\partial^{2} v(x, t)}{\partial t^{2}}+E I \frac{\partial^{4} v(x, t)}{\partial x^{4}}=f(x, t) \quad x \in[0, L], t \in[0, T],
$$

with appropriate boundary and initial conditions, where $v$ is the transversal displacement, $L$ is the length of the beam, $m$ is the mass per unit length, $E$ is the elasticity modulus, $I$ is the area moment of inertia and $f$ is the external force.

To model the inside flow, the plug flow model [14] is used. Let $\mathbf{r}=x \mathbf{i}+y \mathbf{j}$ be a point measured from the origin, where $(\mathbf{i}, \mathbf{j})$ is fixed in a inertial reference, with $\mathbf{j}$ in the transverse direction and $\mathbf{i}$ perpendicular to it, in the axial direction. The velocity of the fluid is given by

$$
\mathbf{v}_{f} \simeq U \mathbf{i}+U \frac{\partial v}{\partial x} \mathbf{j}+\frac{\partial v}{\partial t} \mathbf{j}
$$

where $U$ is the speed of the fluid, and it is assumed that $\partial v / \partial x$ is the (small) rotation of the beam cross sectional area. Hence, the fluid acceleration, which is the material derivative of $\mathbf{v}_{f}$, is given by

$$
\mathbf{a}_{f}=\frac{D \mathbf{v}_{f}}{D t}=\frac{\partial \mathbf{v}_{f}}{\partial t}+\left(\mathbf{v}_{f} \cdot \nabla\right) \mathbf{v}_{f}
$$

If $U$ is constant $d U / d t=0$, then we arrive to

$$
\mathbf{a}_{f}=\left(\frac{\partial^{2} v}{\partial t^{2}}+2 U \frac{\partial^{2} v}{\partial x \partial t}+U^{2} \frac{\partial^{2} v}{\partial x^{2}}\right) \mathbf{j} .
$$

The force per unit length is obtained multiplying the acceleration by the fluid mass per unit length $M_{f}$

$$
\mathbf{f}_{f}=\left(M_{f} \frac{\partial^{2} v}{\partial t^{2}}+2 M_{f} U \frac{\partial^{2} v}{\partial x \partial t}+M_{f} U^{2} \frac{\partial^{2} v}{\partial x^{2}}\right) \mathbf{j}
$$


Including this force in Eq. (1), we arrive in:

$$
\left(m+M_{f}\right) \frac{\partial^{2} v}{\partial t^{2}}+2 M_{f} U \frac{\partial^{2} v}{\partial x \partial t}+M_{f} U^{2} \frac{\partial^{2} v}{\partial x^{2}}+E I \frac{\partial^{4} v}{\partial x^{4}}=f .
$$

The above equation shows that the fluid contributes linearly to the mass, damping and stiffness of the system. Equation $(6)$ is discretized by means of the finite element method: $v^{(e)}(\xi, t)=$ $\mathbf{N}(\xi) \mathbf{u}^{(e)}(t)$, where the shape functions, $\mathbf{N}$, are Hermitian functions and the element displacement vector $\mathbf{u}^{(e)}=\left[\begin{array}{llll}v_{1} & \partial v_{1} / \partial \zeta & v_{2} & \partial v_{2} / \partial \zeta\end{array}\right]$, in which $\xi=x / l_{e}$ is the local coordinate and $l_{e}$ is the element length. The element matrices (mass, fluid damping, fluid stiffness, bending stiffness) are the following: $[M]^{(e)}=\left(m+M_{f}\right) \int_{0}^{1} \mathbf{N}^{T} \mathbf{N} l_{e} d \xi,\left[C_{f}\right]^{(e)}=2 M_{f} U \int_{0}^{1} \mathbf{N}^{T} \mathbf{N}^{\prime} d \xi$, $\left[K_{f}\right]^{(e)}=-M_{f} U^{2} \int_{0}^{1} \mathbf{N}^{\prime T} \mathbf{N}^{\prime} \frac{1}{l_{e}} d \xi,\left[K_{b}\right]^{(e)}=E I \int_{0}^{1} \mathbf{N}_{v}^{\prime \prime T} \mathbf{N}_{v}^{\prime \prime} \frac{1}{l_{e}^{3}} d \xi$. The resulting global discretized system is given by:

$$
[M] \ddot{\mathbf{u}}(t)+\left[C_{f}\right] \dot{\mathbf{u}}(t)+\left(\left[K_{b}\right]+\left[K_{f}\right]\right) \mathbf{u}(t)=\mathbf{f}(t),
$$

where $[M] \in \mathbb{R}^{m \times m}$ is the positive-definite mass matrix, $\left[K_{b}\right] \in \mathbb{R}^{m \times m}$ is the positive-definite bending matrix and $\left[K_{f}\right] \in \mathbb{R}^{m \times m}$ is the negative-definite fluid stiffness matrix. $\mathbf{u}(t) \in \mathbb{R}^{m}$ is the response vector and $\mathbf{f}(t) \in \mathbb{R}^{m}$ is the force vector. Matrix $\left[C_{f}\right] \in \mathbb{R}^{m \times m}$ in general is not symmetric and can be separated in a symmetric positive-definite part and a skew-symmetric part. Due to the boundary conditions of our application, $\left[C_{f}\right]$ is skew-symmetric, however, we still call it damping matrix, even though it not responsible to dissipate energy. In the frequency domain, Eq. (7) can be written as

$$
-\omega^{2}[M] \hat{\mathbf{u}}(\omega)+i \omega\left[C_{f}\right] \hat{\mathbf{u}}(\omega)+\left(\left[K_{b}\right]+\left[K_{f}\right]\right) \hat{\mathbf{u}}(\omega)=\hat{\mathbf{f}}(\omega),
$$

where $\hat{\mathbf{u}}(\omega) \in \mathbb{C}^{m}$ is the response vector and $\hat{\mathbf{f}}(\omega) \in \mathbb{C}^{m}$ is the force vector.

\section{DIMENSIONLESS QUANTITIES}

To be able to do a more general analysis, some dimensionless quantities are introduced:

$$
\begin{array}{lll}
\zeta=\frac{x}{L}, & \eta=\frac{v}{L}, & \tau=t\left(\frac{E I}{\left(m+M_{f}\right) L^{4}}\right)^{1 / 2}, \\
\beta=\frac{M_{f}}{m+M_{f}}, & u=U\left(\frac{M_{f}}{E I}\right), & f=F \frac{L^{3}}{E I}, \\
\varpi & =\frac{1}{\tau} .
\end{array}
$$

where $\zeta$ is the dimensionless length, $\eta$ is the dimensionless transverse displacement, $\tau$ is the dimensionless time, $\beta$ is the ratio of fluid mass, $u$ is the dimensionless speed, $f$ is the dimensionless force, and $\varpi$ is the dimensionless frequency. 


\section{REDUCED-ORDER MODEL}

A reduced-order model [15, 16] is going to be construct for the system given by Eq. [8]. Lets first write the homogeneous equation associated to Eq. (8):

$$
\left(-\omega^{2}[M]+i \omega\left[C_{f}(U)\right]+\left[K_{f}(U)\right]+\left[K_{b}\right]\right) \hat{\mathbf{u}}(\omega)=\mathbf{0},
$$

The following generalized eigenvalue problem is solved to compute the reduction basis that will be used:

$$
\left(-\omega^{2}[M]+\left[K_{b}\right]\right) \boldsymbol{\phi}=\mathbf{0},
$$

corresponding to $U$ equals to zero. With this choice, (1) we guarantee a good basis $([M]$ and $\left[K_{b}\right]$ are positive-definite) and (2) it is not necessary to compute a different basis for each value of $U$. The displacement is written as $\hat{\mathbf{u}}(\omega)=[\Phi] \hat{\mathbf{q}}(\omega)$ and the response in the frequency domain is computed using the reduced-order model:

$$
\left(-\omega^{2}\left[M_{r}\right]+i \omega\left[C_{r}(U)\right]+\left[K_{r}(U)\right]\right) \hat{\mathbf{q}}(\omega)=\hat{\mathbf{f}}(\omega) .
$$

The reduced matrices are given by

$$
\begin{array}{ll}
{\left[M_{r}\right]=[\Phi]^{T}[M][\Phi],} & {\left[C_{r}(U)\right]=[\Phi]^{T}\left[C_{f}(U)\right][\Phi],} \\
{\left[K_{f r}(U)\right]=[\Phi]^{T}\left[K_{f}(U)\right][\Phi],} & {\left[K_{b r}\right]=[\Phi]^{T}\left[K_{b}\right][\Phi],} \\
{\left[K_{r}(U)\right]=\left[K_{f r}(U)\right]+\left[K_{b r}\right],} &
\end{array}
$$

where $[\Phi]=\left[\phi_{1} \phi_{2} \ldots \phi_{n}\right]$, with $(n<m)$. It should be noted that $\left[M_{r}\right]=\delta_{i j}$ and $[\Phi]^{T}\left[K_{b}\right][\Phi]=$ $\delta_{i j} \omega_{i}^{2}$, in which $\delta$ is the Dirac delta.

\section{STABILITY ANALYSIS}

To perform the stability analysis we will double the size of the system in the following way:

$$
\underbrace{\left[\begin{array}{cc}
{[0]} & {[I]} \\
-\left[K_{r}(U)\right] & -\left[C_{r}(U)\right]
\end{array}\right]}_{B_{r}(U)} \underbrace{\left(\begin{array}{c}
\hat{\mathbf{q}} \\
i \omega \hat{\mathbf{q}}
\end{array}\right)}_{\mathbf{y}}=i \omega \underbrace{\left[\begin{array}{cc}
{[I]} & {[0]} \\
{[0]} & {\left[M_{r}\right]}
\end{array}\right]}_{D_{r}}\left(\begin{array}{c}
\hat{\mathbf{q}} \\
i \omega \hat{\mathbf{q}}
\end{array}\right)
$$

Therefore, a generalized eigenvalue problem associated with Eq.(14) is written as:

$$
B_{r}(U) \mathbf{y}=\lambda D_{r} \mathbf{y}
$$

where the eigenvalues $\lambda=\operatorname{Re}(\lambda)+i \operatorname{Im}(\lambda)$, in which $\operatorname{Re}(\cdot)$ and $\operatorname{Im}(\cdot)$ refer to real and imaginary parts. If $\operatorname{Re}(\lambda)>0$ the system is unstable. If $\operatorname{Re}(\lambda)=0$ the system is unstable is the corresponding eigenvalues are not simple. 


\section{PROBABILISTIC MODEL}

To model the uncertainty in the fluid-structure interaction we will make an adaptation of the nonparametric probabilistic approach [9,17] because it is able to cope with model uncertainties. The adaptation consists in using the reduced-order model and the random matrix theory. Since the damping and stiffness matrices come from the same model, they may be statistically correlated and one must find a way to find their joint distribution. In order to achieve this goal, we propose to gather the information of both damping and stiffness of the fluid in a single operator, in the following way:

$$
[A(U)]=i\left[C_{r}(U)\right]+\left[K_{f r}(U)\right]
$$

such that they will be generated randomly together (explained in the sequence) and later we can extract individually the random matrices $\left[\mathbf{C}_{r}(U)\right]$ and $\left[\mathbf{K}_{f r}(U)\right]$ as it will be explained further in this section. The operator needs to pass through some transformations before we can apply the nonparametric probabilistic approach, similar to what was done in [18]. Lets first do the polar decomposition (omitting the dependence on $U$ to simplify the notation):

$$
[A]=[Q][P],
$$

where $[Q]$ is a unitary matrix $\left([Q]^{*}[Q]=[Q][Q]^{*}=[I]\right)$, in which * is the conjugate transpose, and $[P]$ is a Hermitian positive-definite matrix. Note that $[A]$ is invertible for all $U>0$, since $\left[K_{f r}(U)\right]$ is symmetric negative-definite and thus invertible for $U>0$. This decomposition will be computed using the singular value decomposition [19]: $[A]=[U][S][V]^{*}$, with $[P]=$ $[V][S][V]^{*}$ and $[Q]=[U][V]^{*}$. The Cholesky decomposition can be applied to matrix $[P]$ :

$$
[P]=[L]^{*}[L] .
$$

The uncertainty in the fluid-structure interaction model is assumed to be mainly due to the uncertainty on the operator $[P]$, obtained through the polar decomposition. Finally, introducing the random germ $[\mathbf{G}]$ :

$$
[\mathbf{P}]=[L]^{*}[\mathbf{G}][L],
$$

where $[\mathbf{P}]$ and $[\mathbf{G}]$ are random matrices (note that the boldface is used for a random matrix). Thus, we have the random operator

$$
[\mathbf{A}]=[Q][\mathbf{P}]=[Q][L]^{*}[\mathbf{G}][L] .
$$

And the random damping and stiffness matrices happen to be:

$$
\left[\mathbf{C}_{r}\right]=\operatorname{Im}([\mathbf{A}]) \quad \text { and } \quad\left[\mathbf{K}_{f r}\right]=\operatorname{Re}([\mathbf{A}]) .
$$

Without going into further details, the probability density function of the random matrix $[\mathbf{G}]$ and its random generator can be found in [9]. 
The level of uncertainty related to the fluid-structure interaction model is controlled by the dispersion parameter $\delta$ of matrix $[\mathbf{G}]$ defined as:

$$
\delta=\left\{\frac{1}{n} \mathcal{E}\left\{\|[\mathbf{G}]-[I]\|_{F}^{2}\right\}\right\}^{\frac{1}{2}},
$$

where $n$ is the dimension of $[\mathbf{G}],[I]$ is the identity matrix, $\mathcal{E}\{\cdot\}$ denotes the mathematical expectation and $\|[A]\|_{F}=\left(\operatorname{trace}\left\{[A][A]^{T}\right\}\right)^{1 / 2}$ denotes the Frobenius norm. The random eigenvalue problem related to Eq.(14) is given by:

$$
\underbrace{\left[\begin{array}{cc}
{[0]} & {[I]} \\
-\left[\mathbf{K}_{r}(U)\right] & -\left[\mathbf{C}_{r}(U)\right]
\end{array}\right]}_{\mathbf{B}_{r}(U)} \mathbf{Y}=i \Omega \underbrace{\left[\begin{array}{cc}
{[I]} & {[0]} \\
{[0]} & {\left[M_{r}\right]}
\end{array}\right]}_{D_{r}} \mathbf{Y}
$$

where $\left[\mathbf{K}_{r}(U)\right]=\left[\mathbf{K}_{f r}(U)\right]+\left[K_{b r}\right]$, and the random generalized eigenvalue problem that has to be solved is the following:

$$
\mathbf{B}_{r}(U) \mathbf{Y}=\Lambda D_{r} \mathbf{Y}
$$

where the eigenvalues are random variables $\Lambda=\operatorname{Re}(\Lambda)+i \operatorname{Im}(\Lambda)$. Writing $\hat{\mathbf{U}}(\omega)=[\Phi] \hat{\mathbf{Q}}(\omega)$, the stochastic system related to Eq. (12) is given by:

$$
\left(-\omega^{2}\left[M_{r}\right]+i \omega\left[\mathbf{C}_{r}(U)\right]+\left[\mathbf{K}_{r}(U)\right]\right) \hat{\mathbf{Q}}(\omega)=\hat{\mathbf{f}}(\omega),
$$

where $\hat{\mathbf{U}}(\omega)$ is the random response.

\section{NUMERICAL RESULTS}

The beam is divided in 40 finite elements (after convergence check) and the first four normal modes are considered in the analysis. There are two important dimensionless parameters: $\beta$ (mass relation) and $u$ (dimensionless flow speed). In this analysis we fix $\beta=0.24$ and vary the dimensionless speed $u$.

\subsection{Deterministic analysis}

Figure 2 shows the stability charts using the real (Fig. 2(a)) and imaginary parts (Fig. 2(b)) of half of the eigenvalues. As $u$ increases, the system gets more unstable. When $u=3.15$ there is the first divergence mode, when $u=6.26$ there is the second divergence mode and the coupled-mode flutter, and when $u=9.43$ there is the second divergence mode, as indicated in the figure. Figure 2(b) shows that as $u$ increases, the system gets less stiff, that is the four natural frequencies decrease. These results are in accordance with the ones found in [14].

Figure 3 shows the amplitude of the displacement in the frequency domain for $u=\{1.0,2.5\}$. The curve related to the higher $u$ is moved to the left (system less stiff). The force applied was $f=1 \times 10^{-4}$ at each degree of freedom of the beam and the response shown is the absolute value of the dimensionless displacement in the middle of the beam.

The next section analyzes how these results change when the stochastic model is taken into account. 


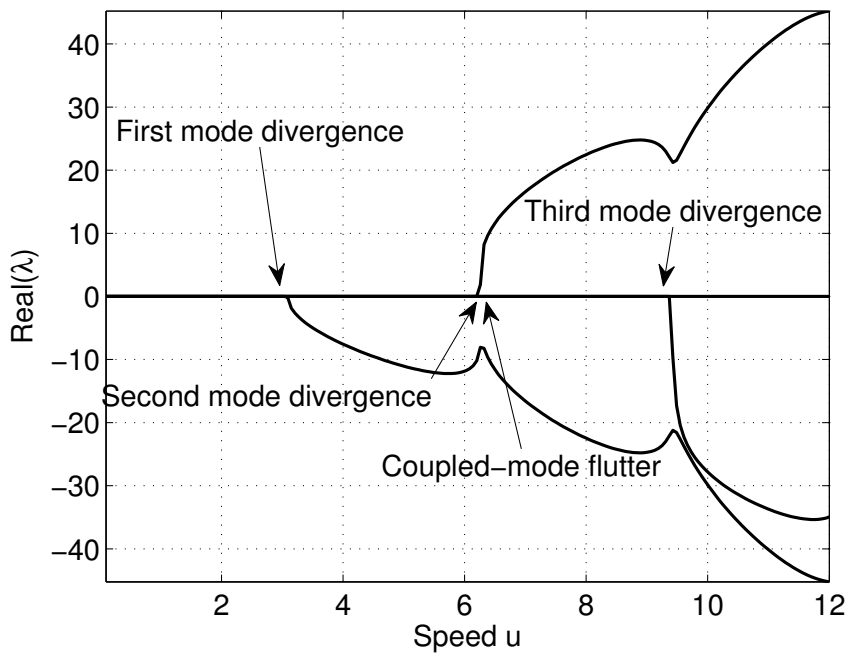

(a)

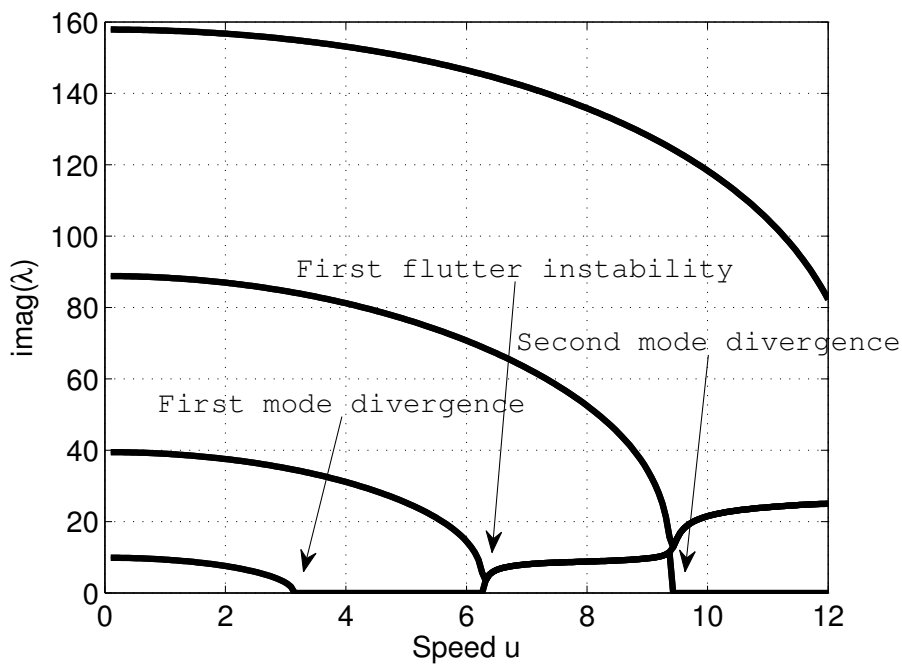

(b)

Figure 2: Stability charts. Real (a) and imaginary part (b) of $\lambda$.

\subsection{Stochastic analysis}

Now we analyze the stochastic response of the system. We have used 1000 Monte Carlo samples in the analysis, since it is sufficient for a reasonable mean square convergence of the random eigenvalues. Figures 4 and 5 show the 95\% confidence region for $\delta=\{0.05 ; 0.1\}$. When $\delta$ increases the confidence region also increases, as expected. It can be seen that when $u=0$ there is no uncertainty in $\lambda$, but the uncertainty then increases. However the robustness of the system varies depending on the speed $u$, as it is observed for $\operatorname{Re}(\lambda)$; between $u=7$ and 8 the confidence region is thinner and close to $u=12$ it is wider.

When the lines of the graphics cross each other, it is hard to follow the modes. Instead of analyzing these crossings, lets evaluate the probability of being in a instability region. We define two failure probabilities: (1) one related to the divergence instability and (2) other related to the coupled-mode flutter instability. Figure 6(a) shows that until about $u=3$ the probability of divergence is close to zero, but for $u>3.5$ it becomes $100 \%$. The same way, Figure 6(b) shows that until about $u=5$ the probability of coupled-mode flutter is close to zero, but for $u>6.5$ it becomes $100 \%$. To get a better feeling of this probability of failure, take a look at Figure 7. It 


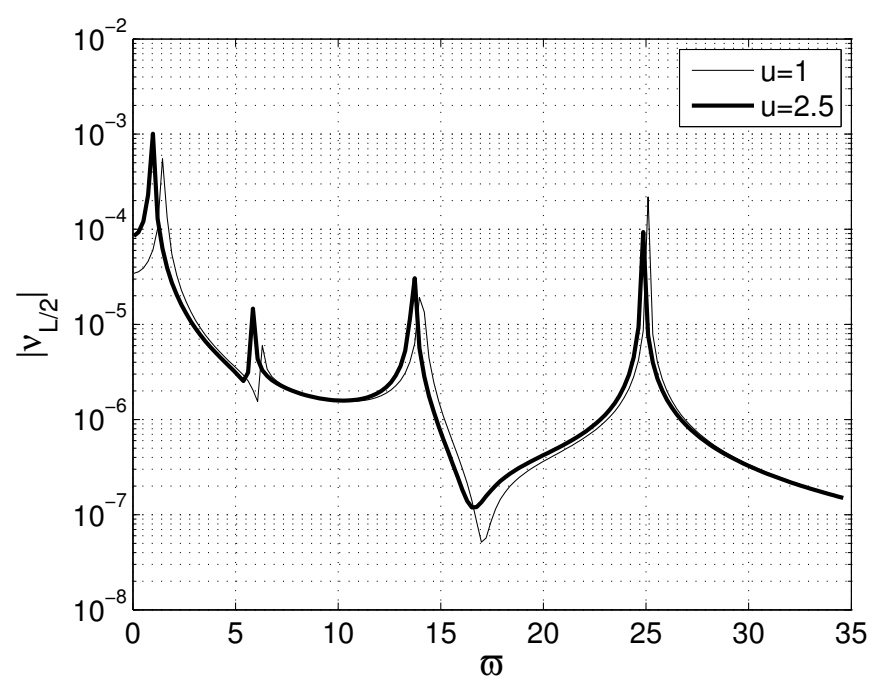

Figure 3: Frequency response amplitude at $\zeta=0.5$.

shows the histogram of $\max (\operatorname{Re}(\Lambda))$ related to the coupled-mode flutter for two different $u$ 's. In both cases there is a big probability that $\max (\operatorname{Re}(\Lambda))=0$. However, for higher flow speeds, the probability that this random variable is greater than zero increases. An interesting fact to note is that the random variable $\max (\operatorname{Re}(\Lambda))$ follows a mixed distribution, with a concentrated probability at point zero (stable condition, if only coupled-mode flutter instability is considered) and a continuous distribution above zero (unstable condition relate to flutter).

Now we will detail the analysis of the probability of being in a coupled-mode flutter condition for different levels of uncertainties. Figure 8 shows the probability of coupled-mode flutter as a function of the flow speed $u$ for different levels of uncertainty (delta values). The dashdoted arrows show that if the deterministic $(\delta=0)$ limit speed is considered, the probability of failure would be about 50\%; which is a bad scenario. This is an important result, and it shows that an uncertainty model should be taken into account.

On the other hand, the solid arrows show that if we define a failure probability, say $20 \%$, depending on the level of uncertainty there will be a limit flow speed for each value of delta: $u=6.12$ for $\delta=0.1, u=6.20$ for $\delta=0.05, u=6.20$ for $\delta=0.05$ and $u=6.26$ for $\delta=0.01$. As expected, if delta increases, the limit $u$ should decrease because the scenario is getting worse. Finally, Figure 9 shows the response in the frequency domain for $u=3.1$ and $\delta=0.1$. It is noted that the uncertain region is bigger when the frequency is close to zero, where the stabilities are put in check.

\section{CONCLUDING REMARKS}

The problem of a tube with internal flow is analyzed in this paper. A probabilistic model based on the nonparametric probabilistic approach was proposed to model uncertainties in the fluid-structure interaction. This probabilistic model is able to take into account modeling errors related to the fluid-structure interaction.

A simple Euler-Bernoulli beam and a plug flow model are considered to model the system, which is discretized by means of the finite element methods and reduced using the normal modes of the beam. The stability analysis is done for different levels of uncertainties showing how confidence limits change for different values of dimensionless flow speed. Also, the probability of instability is computed for different levels of uncertainty of the fluid-structure 


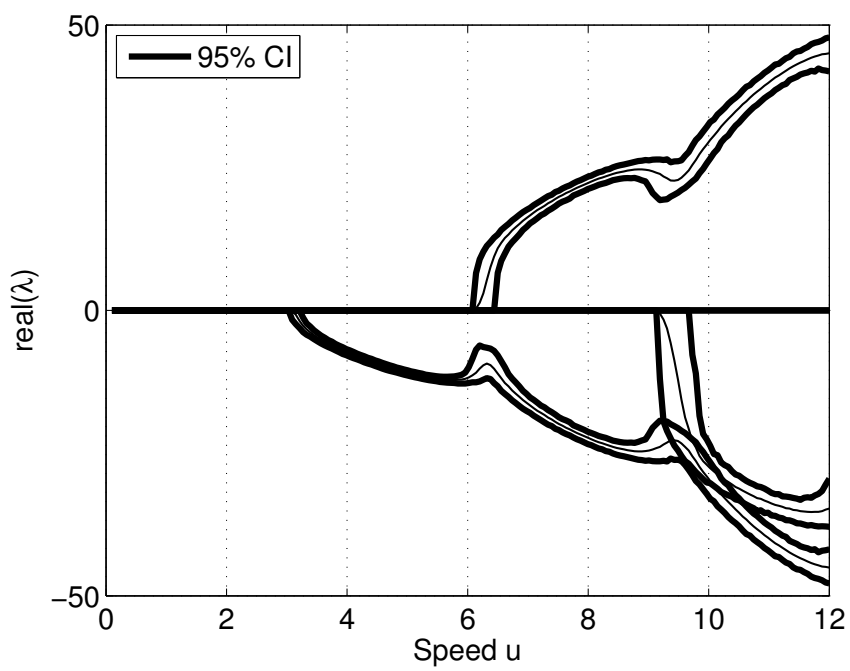

(a)

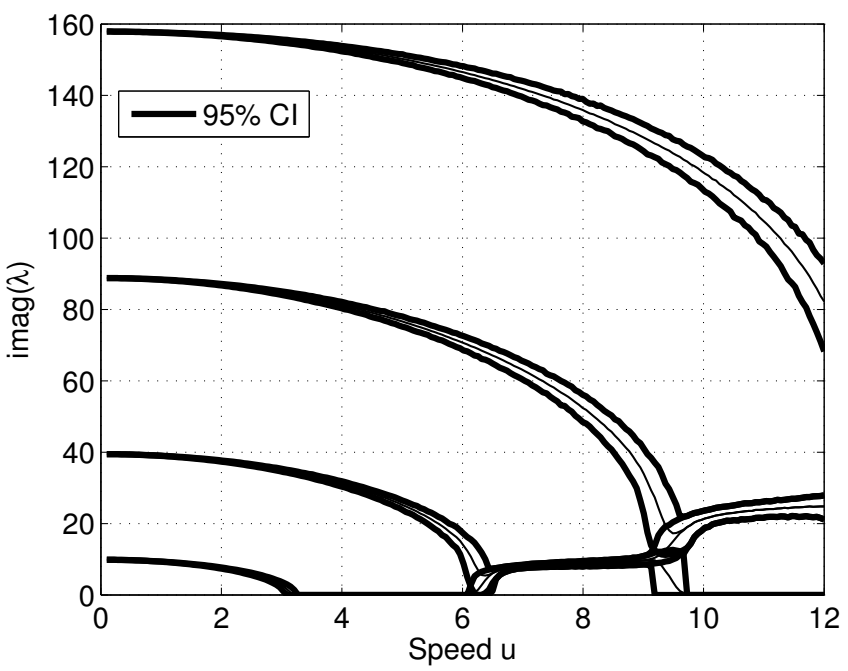

(b)

Figure 4: $95 \%$ confidence interval for $\delta=0.05$

interaction. An interesting fact is that the random variable related to the max of the real part of the random eigenvalues has a mixed distribution (discrete and continuous). It seems that the proposed probabilistic model is well suited for the problem analyzed, although other analysis should be performed and the previsions compared with experimental data.

\section{Acknowledgements}

The authors are grateful to the financial support from the Brazilian agencies: CAPES, CNPq, and FAPERJ. And specifically the project CAPES-COFECUB.

\section{REFERENCES}

[1] T. G. Ritto, C. Soize and R. Sampaio, Nonlinear dynamics of a drill-string with uncertain model of the bit-rock interaction. International Journal of Non-Linear Mechanics, 44(8), 865-876, 2009.

[2] S. Rinaldi, S. Prabhakar, S. Vengallatore and M.P Paidoussis, Dynamics of microscale 


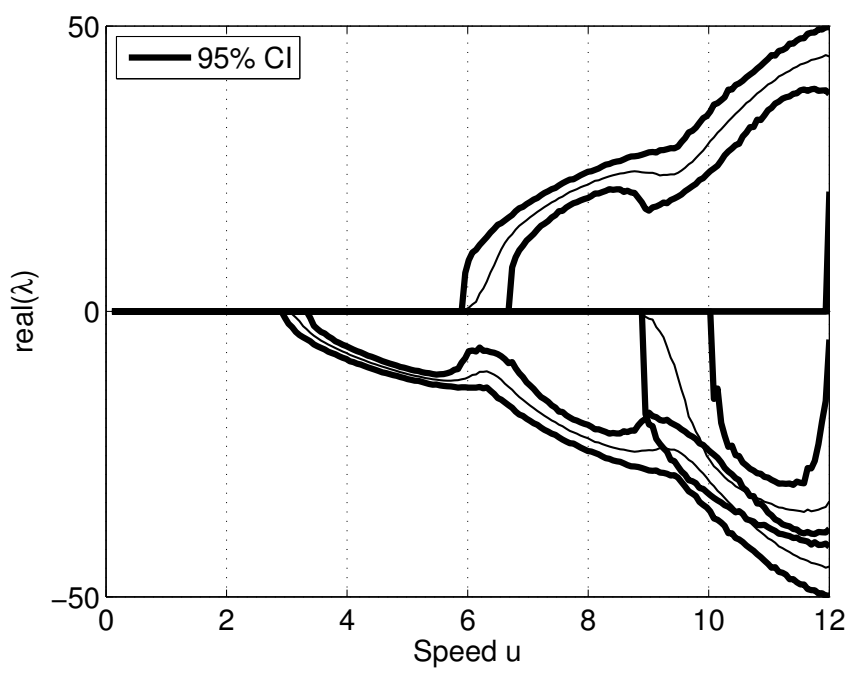

(a)

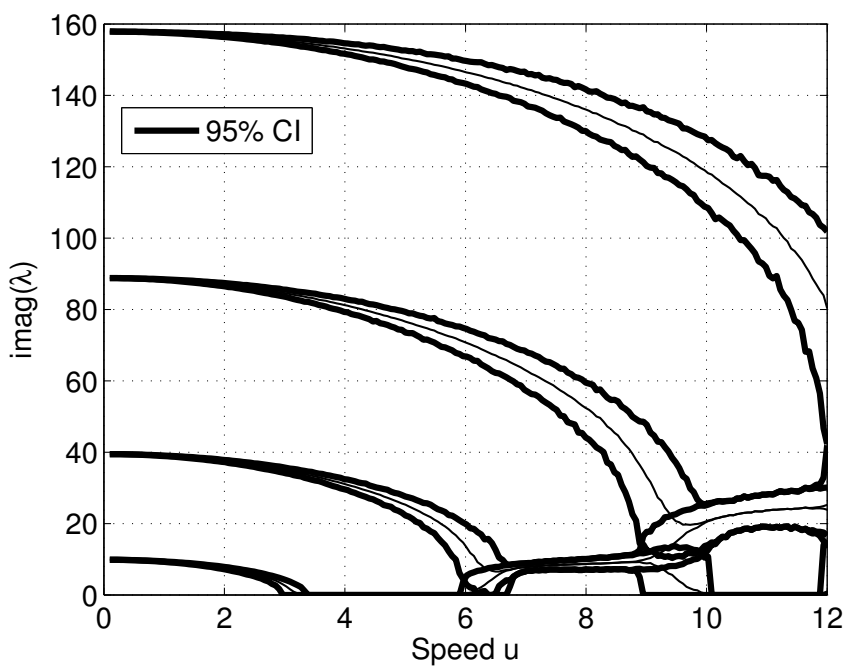

(b)

Figure 5: 95\% confidence interval for $\delta=0.1$.

pipes containing internal fluid flow: Damping, frequency shift, and stability. Journal of Sound and Vibration, 329(8), 1081-1088, 2010.

[3] P. Soltani, M.M. Taherian and A. Farshidianfar, Vibration and instability of a viscous-fluidconveying single-walled carbon nanotube embedded in a visco-elastic medium. Journal of Physics D: Applied Physics, 43(42), art. no. 425401, 2010.

[4] Ritto, T; Sampaio, R.; Rochinha, F.; Model uncertainties of flexible structures vibrations induced by internal flows. Journal of the Brazilian Society of Mechanical Sciences and Engineering, 33, 373-380, 2011.

[5] A. H. Nayefh and B. Balachandran, Applied Nonlinear Dynamics, Wiley Series in Nonlinear Science, 1995.

[6] G. I. Schueller, H. J. Pradlwarter and P. S. Koutsourelakis, A critical appraisal of reliability estimation procedures for high dimensions. Probabilistic Engineering Mechanics, 19(4), 463-474, 2004. 


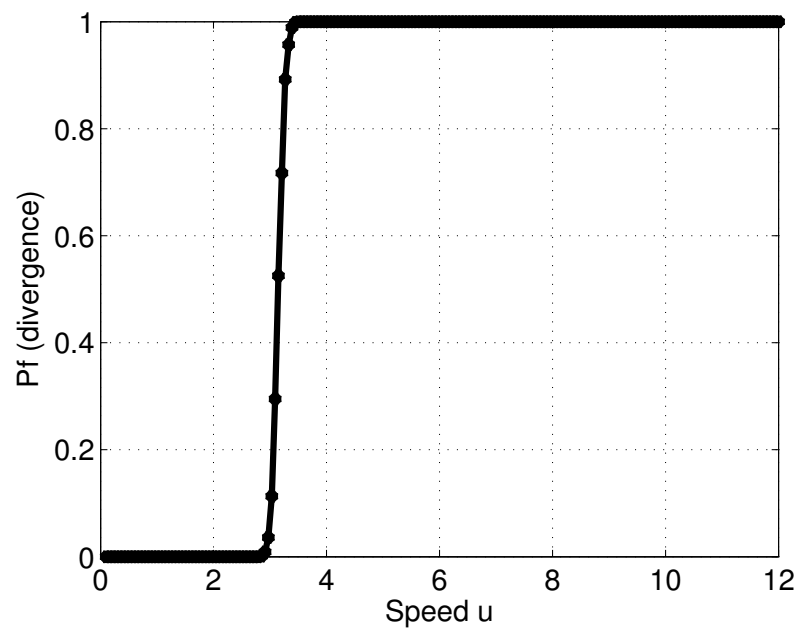

(a)

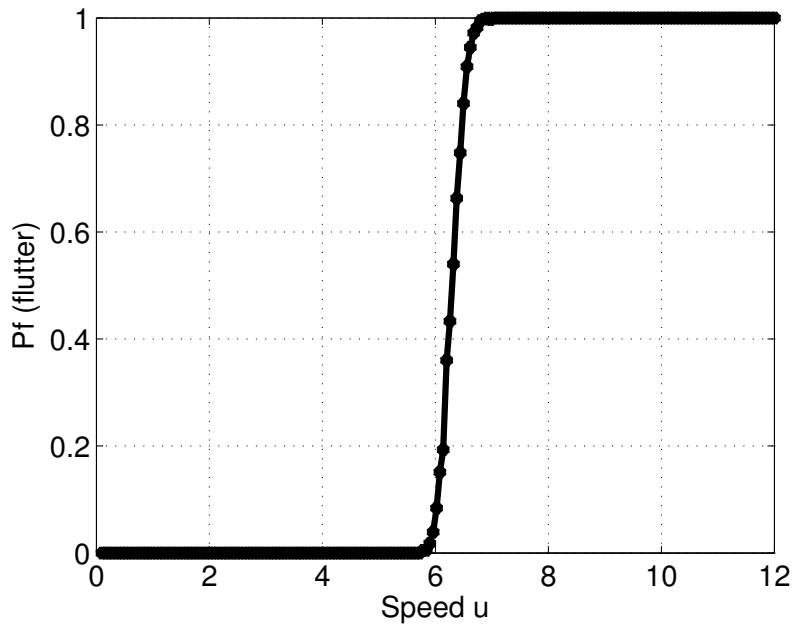

(b)

Figure 6: For different values of $u$ and $\delta=0.1$., (a) probability of being in a divergence region and (b) probability of being in a ccoupled-mode flutter region.

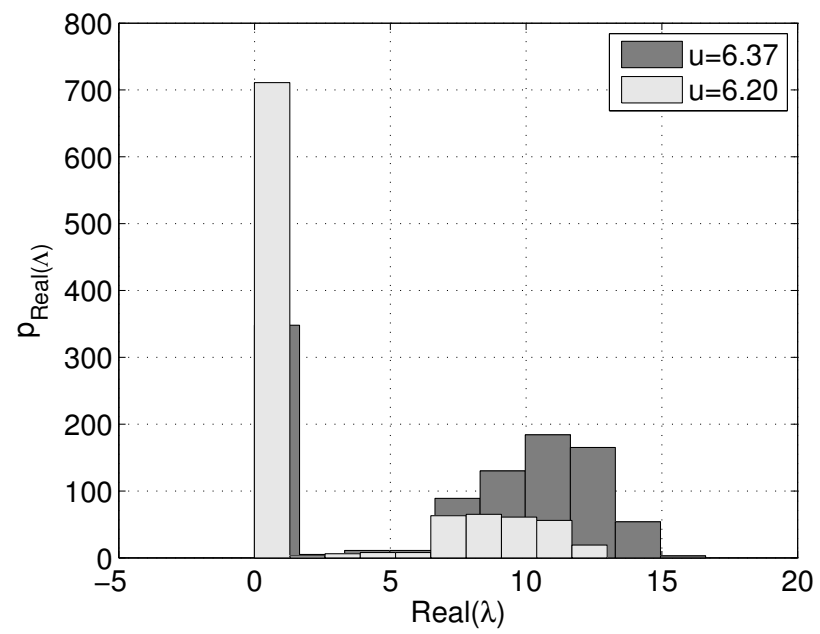

Figure 7: Histogram of $\max (\operatorname{Re}(\Lambda))$ for two different $u$ 's, considering coupled-mode flutter mode instability. 


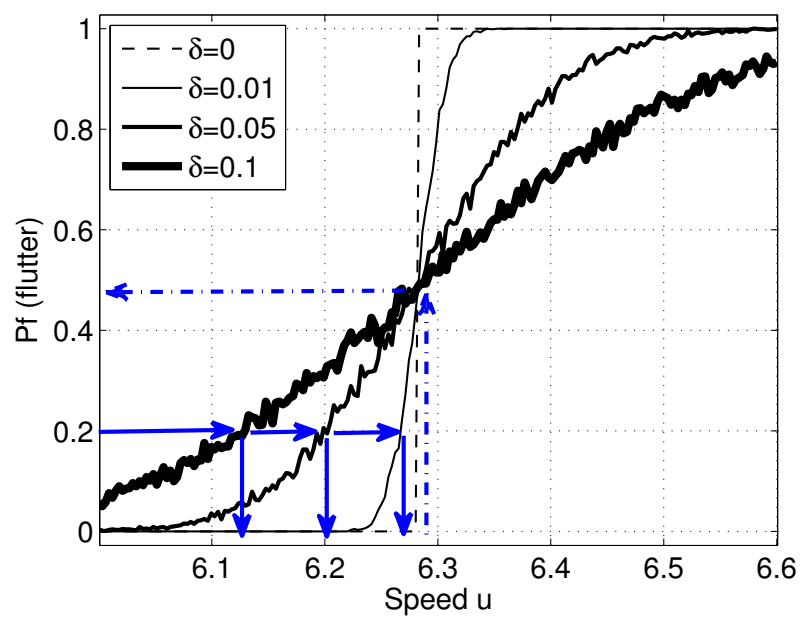

Figure 8: For different values of $u$ and $\delta$, probability of being in a coupled-mode flutter region.

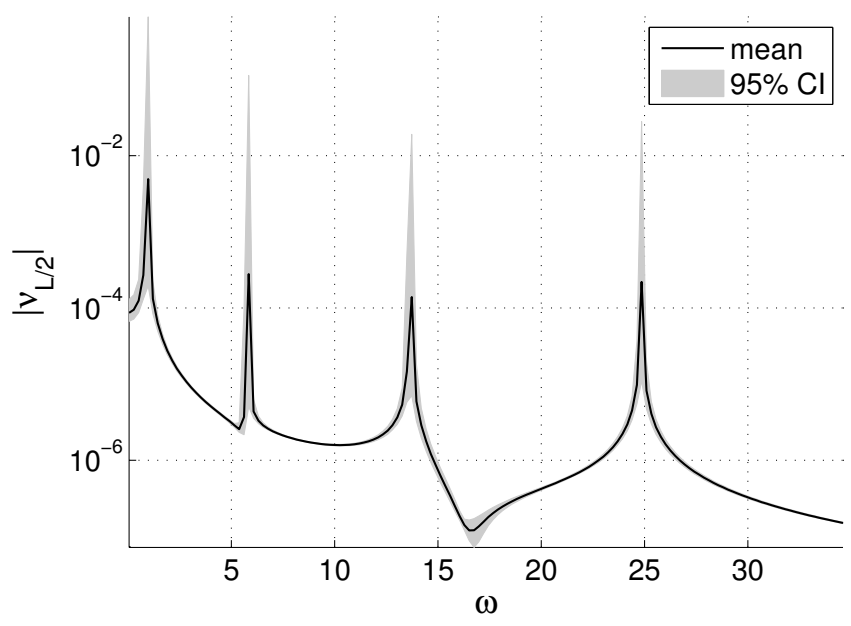

Figure 9: Frequency response amplitude at $\zeta=0.5$ for $u=2.5$ and $\delta=0.1$.

[7] M.P. Paidoussis and N. T. Issid, Dynamic stability of pipes conveying fluid . Journal of Sound and Vibration, 33(3), 267-294, 1974.

[8] Y. K. Lin and G. Cai, Probabilistic Structural Dynamics, McGraw-Hill, 1995.

[9] C. Soize, A nonparametric model of random uncertainties for reduced matrix models in structural dynamics. Probabilistic Engineering Mechanics, 15, 277-294, 2000.

[10] J. F Durand, C. Soize and L. Gagliardini, Structural-acoustic modeling of automotive vehicles in presence of uncertainties and experimental identification and validation. J. Accoust. Soc. Am., 124(3), 1513-1525, 2008.

[11] C. Chen, D. Duhamel and C. Soize, Probabilistic approach for model and data uncertainties and its experimental identification in structural dynamics: Case of composite sandwich panels. Journal of Sound and Vibration, 194(1-2), 64-81, 2006.

[12] E. Jaynes, Probability Theory: The Logic of Science, Cambridge University Press, 2003. 
[13] D. J. Inman, Engineering Vibration, 4th Edition, Prentice-Hall, Inc., 2007.

[14] M. P. Paidoussis, Fluid-Structure Interactions: Slender Structures and Axial Flow, Vol. 1, Academic Press, 1998.

[15] J. M. Crolet and R. Ohayon, Computational Methods for Fluid-Structure Interaction, John Wiley \& Sons Inc., 1994.

[16] Z.-Q.S. Qu, Model Order Reduction Techniques: with Applications in Finite Element Analysis, Springer, 2010.

[17] C. Soize, Random matrix theory for modeling uncertainties in computational mechanics. Computer Methods in Applied Mechanics and Engineering, 194(12-16), 1333-1366, 2005.

[18] M. Mbaye and C. Soize, J.-P. Ousty and E.Capiez-Lernout, Robust analysis of design in vibration of turbomachines. ASME Journal of Turbomachinery, 135(2), art. no. 021008, 2013.

[19] G. H. Golub and C. F. Van Loan, Matrix Computations, 3rd Edition, Johns Hopkins, 1996. 